\title{
ASSESSING EFFECTS OF NEAR-FIELD SYNERGISTIC LIGHT ABSORPTION ON ORDERED INORGANIC PHOTOTROPIC GROWTH
}

\author{
AZHaR I. CARIM ${ }^{\dagger, \S}$, MAdEline C. MEIER $^{\dagger, \S}$, KATHLEEN M. KenNEDY $^{\ddagger}$, MATTHIAS H. \\ RICHTER $^{\ddagger}$, KATHRYN R. HAMANN ${ }^{\dagger}$ AND NATHAN S. LEWIS ${ }^{\dagger, *}$ \\ ${ }^{\dagger}$ Division of Chemistry and Chemical Engineering \\ Division of Engineering and Applied Sciences \\ California Institute of Technology \\ Pasadena, CA 91125
}




\section{S1. Contents}

This document contains a description of the experimental and computational methods utilized in this work (Sections S2 and S3), additional computational data (Section S4), and a list of associated references (Section S5). 


\section{S2. Experimental Methods}

Materials and Chemicals Acetone (99.5 \%, EMD Millipore), isopropanol (99.5 \%, VWR), methyl isobutyl ketone (90 - $100 \%$, J.T.Baker), poly(methyl methacrylate) (PMMA, 495 A8, Microchem), buffered oxide etchant ( $\mathrm{NH}_{4} \mathrm{~F}: \mathrm{HF}$ in a $6: 1$ volume ratio, Transene), In (99.999\%, Alfa Aesar), Ga (99.999 \%, Alfa Aesar), $\mathrm{H}_{2} \mathrm{SO}_{4}$ (ACS Reagent, J.T.Baker), $\mathrm{SeO}_{2}$ (99.4 \%, Alfa Aesar), and $\mathrm{TeO}_{2}\left(99+\%\right.$, Sigma-Aldrich) were used as received. $\mathrm{H}_{2} \mathrm{O}$ with a resistivity $\geq 18.2$ $\mathrm{M} \Omega \mathrm{cm}$ (Barnstead Nanopure System) was used throughout. $\mathrm{n}^{+}-\mathrm{Si}(100)(0.001-0.0035 \Omega \mathrm{cm}$, Asdoped, $525 \pm 15 \mu \mathrm{m}$ thick, single-side polished, Addison Engineering) was used as a substrate for deposition. Flash-Dry Ag Paint (SPI Supplies), EP21ARHTND Epoxy (MasterBond) and nitrocellulose-based nail polish were used to assemble the working electrodes.

Substrate Preparation $\mathrm{n}^{+}-\mathrm{Si}$ wafers were first rinsed with acetone and isopropanol. PMMA resist was then applied by spin-coating for $1.0 \mathrm{~min}$ to effect a $0.8 \mu \mathrm{m}$ thick layer and then cured for $5.0 \mathrm{~min}$ at $180^{\circ} \mathrm{C}$. Patterns consisting of circles with diameters of $0.2,0.4,0.8,1.2$, and 1.6 $\mu \mathrm{m}$, on a square lattice with a pitch of $10 \mu \mathrm{m}$, were designed using AutoCAD (Autodesk) and fractured using BEAMER (GenISys). A Raith EBPG 5000+ electron-beam pattern generator was used to write the patterns into the resist with a $100 \mathrm{kV}$ accelerating voltage, $300 \mu \mathrm{m}$ aperture, $5 \mathrm{nA}$ current, and a dose of $1000 \mu \mathrm{C} \mathrm{cm}^{-2}$. The pattern was then developed by immersing the substrates in a mixture of methyl isobutyl ketone : isopropanol in a $1: 3$ volume ratio for $1.0 \mathrm{~min}$ at room temperature, producing an array of circular well structures. The patterned wafers, along with additional unpatterned $\mathrm{Si}$, were then cut into square $0.40 \mathrm{~cm}$ by $0.40 \mathrm{~cm}$ sections using a Dynatex GST-150 scriber-breaker system; these square pieces were then used as substrates for the photoelectrochemical growth of Se-Te. 
Electrode Preparation One end of a Sn-coated Cu wire (22 AWG) was bent to form a small, flat coil and the wire was threaded through glass tubing $(6 \mathrm{~mm} \mathrm{O}$. D.) such that the coil was just outside the tubing. Epoxy was applied to seal the end of the tube from which the coil protruded. A eutectic mixture of Ga and In was scratched with a carbide-tipped scribe into the unpolished back surfaces of the Si sections. The wire coil was then contacted to the unpolished surface and affixed with Ag paint. Nail polish was applied to insulate the unpolished face, the wire-coil contact, and the exposed wire between the coil and epoxy seal. Immediately before deposition, the Si portion of the electrode was immersed in buffered oxide etchant for $\sim 45 \mathrm{~s}$ to remove surficial $\mathrm{SiO}_{\mathrm{x}}$ from the $\mathrm{Si}$. The electrode was then rinsed with $\mathrm{H}_{2} \mathrm{O}$ and dried under a stream of $\mathrm{N}_{2}(\mathrm{~g})$.

Electrode Illumination Photoelectrochemical depositions were performed using a narrowband light-emitting diode (LED) source (Thorlabs SOLIS-940C) with an intensity-weighted average wavelength, $\lambda_{\text {avg, }}$ of $934 \mathrm{~nm}$ and a spectral bandwidth (FWHM) of $55 \mathrm{~nm}$. The output of the diode source was collected, condensed, and collimated using a series of three lenses: an aspheric lens $(\varnothing 25.4 \mathrm{~mm}, \mathrm{f}=16 \mathrm{~mm})$, followed by a bi-convex lens $(\varnothing 50.8 \mathrm{~mm}, \mathrm{f}=16 \mathrm{~mm})$, and last a plano-convex lens $(\varnothing 50.8 \mathrm{~mm}, \mathrm{f}=100 \mathrm{~mm})$. A film polarizer (Thorlabs LPNIRE200-B) was inserted after the lenses to produce vertical linear polarization. A 120 grit ground-glass (N-BK7) diffuser was placed immediately in front of the photoelectrochemical cell to ensure spatial homogeneity of the illumination. The light intensity incident on the electrode was measured by placing a calibrated Si photodiode (Thorlabs FDS100), instead of an electrode assembly, in the photoelectrochemical cell with electrolyte, and the steady-state current response of the $\mathrm{Si}$ photodiode was measured. Depositions were performed with a light intensity of $195 \mathrm{~mW} \mathrm{~cm}^{-2}$.

Photoelectrochemical Deposition Photoelectrochemical deposition was performed using a Bio-Logic SP-200 potentiostat. Deposition was performed in a glass cell with a quartz window. A 
three-electrode, two-compartment cell was used with an Ir wire counter electrode (99.999 \%, Sigma-Aldrich) isolated behind a porous glass frit, and a $\mathrm{Ag} / \mathrm{AgCl}$ reference electrode $(3 \mathrm{M} \mathrm{KCl}$, Bioanalytical Systems). Films were deposited from an aqueous solution of $0.0200 \mathrm{M} \mathrm{SeO}_{2}, 0.0100$ $\mathrm{M} \mathrm{TeO}_{2}$, and $2.00 \mathrm{M} \mathrm{H}_{2} \mathrm{SO}_{4}$. Deposition was effected by biasing the patterned $\mathrm{Si}$ electrode, illuminated as described above (Electrode Illumination), potentiostatically at $-0.400 \mathrm{~V}$ vs. $\mathrm{Ag} / \mathrm{AgCl}$ for $3.00 \mathrm{~min}$ at room temperature. After deposition, the electrode was immediately removed from the cell, rinsed with $\mathrm{H}_{2} \mathrm{O}$, and then dried under a stream of $\mathrm{N}_{2}(\mathrm{~g})$. The patterned substrate with the top-facing Se-Te deposit was mechanically separated from the rest of the electrode assembly. The nitrocellulose-based insulation and the majority of the Ag paint and InGa eutectic were then removed mechanically. Patterned substrates were then immersed in acetone for $\sim 5$ min to remove the PMMA resist, rinsed with isopropanol, and then dried under a stream of $\mathrm{N}_{2}(\mathrm{~g})$.

Microscopy Scanning-electron micrographs (SEMs) were obtained with a FEI Nova NanoSEM 450 at an accelerating voltage of $5.00 \mathrm{kV}$ with a working distance of $5 \mathrm{~mm}$ and an inlens secondary electron detector. Micrographs were acquired with a resolution of 688 pixels $\mu \mathrm{m}^{-1}$ over $2 \mu \mathrm{m}^{2}$ areas. 


\section{S3. Computational Methods}

Growth Modeling The growths of the photoelectrochemically deposited films were simulated with an iterative growth model in which electromagnetic simulations were first used to calculate the local photocarrier-generation rates at the film surface. Then, mass addition was simulated via a Monte Carlo method wherein the local photocarrier-generation rate weighted the local rate of mass addition along the film surface.

Simulations of confined growth began with a semi-infinite Si substrate with an atop PMMA layer with a single well structure that had the same dimensions as the wells used in the experiments described in Section S2. In the first step, the light-absorption profile under a linearly polarized, plane-wave illumination source was calculated using full-wave finite-difference time-domain (FDTD) simulations. Perfectly matched layer boundary conditions were utilized at the boundaries of the confined growth FDTD simulations. In the second step, a Monte Carlo simulation was performed in which mass was added to the upper surface of the structure with a probability $F$ :

$$
F(G)=G \prod_{i=1}^{3} \frac{x_{i}}{r_{i}} \quad(\text { Equation 1) }
$$

where $G$ is the spatially dependent photocarrier-generation rate at the deposit/solution interface, $x_{i}$ is the fraction of $i^{\text {th }}$ nearest neighbors occupied in the cubic lattice, and $r_{i}$ is the distance to the $i^{\text {th }}$ nearest neighbor. The multiplicative sum in the definition of this probability (Equation 1) serves to reduce the surface roughness of the film, to mimic the experimentally observed surface roughness. After the initial Monte Carlo simulation, the absorbance of the new, structured film was then calculated in the same manner as for the initial planar film, and an additional Monte Carlo simulation of mass addition was performed. This process of absorbance calculation and mass addition was repeated for a total of 30 iterations. The amount of mass added in each Monte Carlo simulation equaled that of a $15 \mathrm{~nm}$ planar layer covering the projected well area, preventing 
optically-large changes in the feature dimensions between iterations and thus enabling a discretized computational approximation of the continuous growth process.

Optical Simulations of Simplified Morphologies Three-dimensional FDTD simulations were utilized to calculate the profiles of light absorption and the time-average electric-field magnitude for simplified lamellar structures. A single plane-wave source polarized with the electric field parallel to the lamellar long axis was considered. Periodic boundary conditions were imposed in the direction parallel to the lamellar long axis whereas perfectly matched layer boundary conditions were imposed in each of the perpendicular directions.

Dipole Emission Simulations FDTD simulation of dipole emission was utilized to model the interfacial optical modulation at the onset of growth. A two-dimensional square simulation plane was utilized. Dipoles were arranged with separation of twice the emission wavelength and the oscillation axis was set perpendicular to the separation axis. Perfectly matched layer boundary conditions were imposed in both directions.

General Parameters All FDTD simulations were performed with FDTD Solutions (Lumerical). Previously measured values of the complex refractive index of Se-Te were utilized. Values of $n=1.33$ and 1.48 were used for the refractive index of the electrolyte and the PMMA, respectively. ${ }^{1,2}$ The simulations utilized the intensity-weighted average wavelength, $\lambda_{\text {avg, }}$ of the experimental source described in Section S2 and were discretized using a uniform threedimensional cubic mesh with lattice constants of $10 \mathrm{~nm}$ and $2 \mathrm{~nm}$ for the growth modeling and simplified simulations, respectively. 


\section{S4. Additional Computational Data}

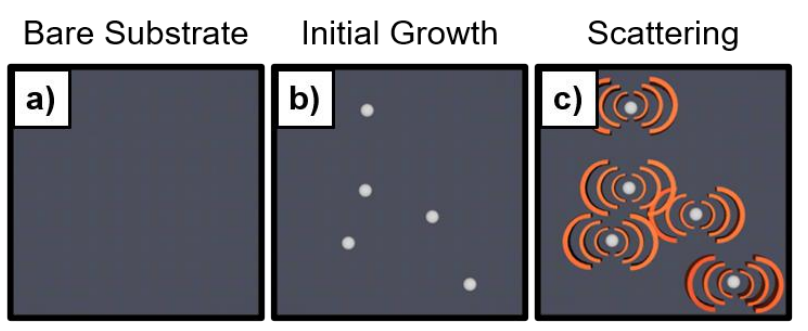

Figure S1. Origin of structure genesis via inorganic phototropic growth on a bare, isotropic substrate (a). Electrochemical deposition produces a surface topology (b) that scatters the incident light beam (c).

Inorganic phototropic growth was effected by light-mediated electrochemical synthesis of Se-Te using vertically polarized illumination with an intensity-weighted average wavelength $\left(\lambda_{\text {avg }}\right)$ of $934 \mathrm{~nm}$ from an incoherent, narrowband light-emitting diode source. Light absorption in the deposited Se-Te material accelerates the rate of electrochemical growth. ${ }^{3}$ The surface topology generated by the initial deposition results in scattering of the incident illumination; this initial process is graphically summarized in Figure S1. This scattering in turn modulates the electric field amplitude at the growth interface. The structure of the interfacial electric field defined by scattering of the vertically polarized illumination was calculated by modeling the scatterers as point dipole emission sources.

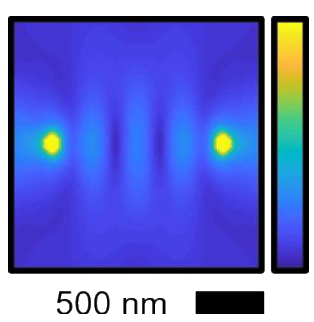

Figure S2. Normalized time-average of the E-field magnitude from two dipoles emitting radiation with a free space wavelength of $\lambda=934 \mathrm{~nm}$ in a medium of refractive index $n=1.33$. Dipoles separated by a distance of twice the wavelength along the horizontal with the oscillation axis perpendicular to the axis of separation.

Figure S2 presents a simulation of the normalized time-averaged electric field magnitude from two dipoles emitting radiation with a free space wavelength of $\lambda=934 \mathrm{~nm}$ in a medium of refractive index $n=1.33$ (reflective of the growth solution) and separated by a distance of two wavelengths 
perpendicular to the oscillation axis. Periodic bands of elevated field intensity were observed. Light-driven electrochemical growth occurs preferentially in these areas of localized field enhancement. Thus, these data are consistent with the anisotropic, ordered morphology observed experimentally (Figure 1a) and present a basis for inorganic phototropic growth.
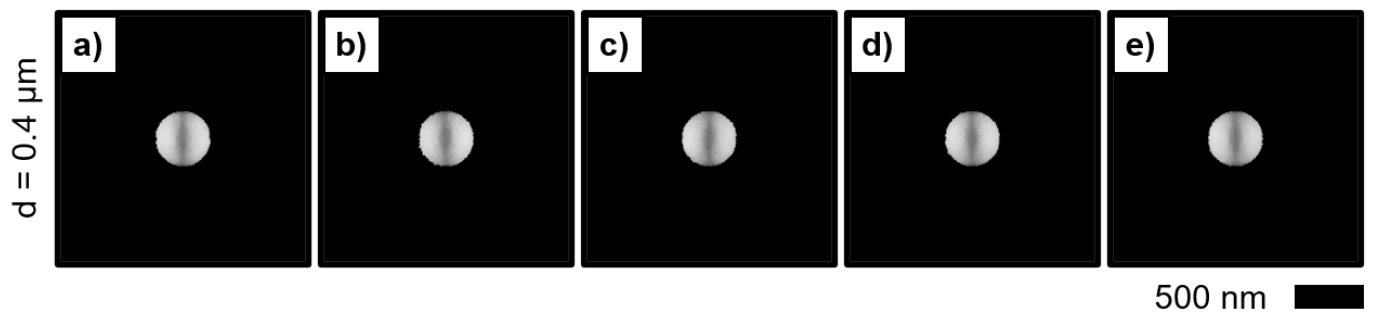

Figure S3. Simulated Se-Te deposit morphologies generated considering $\lambda_{\text {avg }}=934 \mathrm{~nm}$ illumination with areal constraint to the circular diameter $d=0.4 \mu \mathrm{m}$. Each panel presents the result of a fully independent set of growth computations.

Figure S3 presents simulated morphologies of Se-Te deposits generated from independent sets of computations using the iterative, two-step growth model considering $\lambda_{\text {avg }}=934 \mathrm{~nm}$ illumination with areal constraint to the circular diameter $d=0.4 \mu \mathrm{m}$. The extremely close accord between these mutually independent simulations of bottom-up mass addition compellingly indicates that the observed structures are a direct result of light-matter interactions between the input illumination and the evolving Se-Te deposits. 


\section{S5. References}

(1) Hale, G. M.; Querry, M. R. Optical Constants of Water in the 200-nm to 200- $\mu$ m Wavelength Region. Appl. Opt. 1973, 12, 555-563.

(2) Zhang, X.; Qiu, J.; Li, X.; Zhao, J.; Liu, L. Complex refractive indices measurements of polymers in visible and near-infrared bands. Appl. Opt. 2020, 59, 2337-2344.

(3) Meier, M. C.; Cheng, W.-H.; Atwater, H. A.; Lewis, N. S.; Carim, A. I. Inorganic Phototropism in Electrodeposition of Se-Te. J. Am. Chem. Soc. 2019, 141, 18658-18661. 